\title{
Adaptive Sliding Mode Control for a Class of Manipulator Systems with Output Constraint
}

\author{
Guangshi Li $\mathbb{D}$ \\ Computer Center, Anshan Normal University, Anshan 114016, China \\ Correspondence should be addressed to Guangshi Li; wslgs@netease.com
}

Received 19 December 2020; Revised 19 January 2021; Accepted 25 January 2021; Published 3 February 2021

Academic Editor: Qiuye Sun

Copyright (c) 2021 Guangshi Li. This is an open access article distributed under the Creative Commons Attribution License, which permits unrestricted use, distribution, and reproduction in any medium, provided the original work is properly cited.

\begin{abstract}
In this paper, an adaptive sliding mode control method based on neural networks is presented for a class of manipulator systems. The main characteristic of the discussed system is that the output variable is required to keep within a constraint set. In order to ensure that the system output meets the time-varying constraint condition, the asymmetric barrier Lyapunov function is selected in the design process. According to Lyapunov stability theory, the stability of the closed-loop system is analyzed. It is demonstrated that all signals in the resulted system are bounded, the tracking error converges to a small compact set, and the system output limits in its constrained set. Finally, the simulation example is used to show the effectiveness of the presented control strategy.
\end{abstract}

\section{Introduction}

The application of manipulator is of great significance to the development of human society. So far, many effective control strategies have been produced for manipulator control, such as the PID control $[1,2]$, the robust control [3-5], and the sliding mode control [6-8]. Among them, the PID control is more suitable for the control theory of other technologies difficult to adopt, or the mathematical models are not more accurate. In [9], a new adaptive neural network control method, which is based on a simple structure-like PID control, is constructed to deal with the robot system with incomplete model and disturbance. The feature of the robust control is that once the controller is designed, its parameters are fixed; that is, it is not affected by external interference, and it can guarantee the control performance. In $[10,11]$, the robust control approaches are proposed to solve the position tracking problem of the manipulator systems. However, if the function of the considered system needs to be improved or made major changes, then the controller requires to be redesigned, which cannot be realized for the robust control method. The sliding mode control has the good stability against external disturbance and system unmodeled dynamics. For this, the sliding mode control will be considered in this paper.
In recent years, with the rapid development of science and technique, the adaptive control technology has become particularly important. Especially, the fuzzy logic systembased and neural network-based adaptive control is one of the current research hot topics. These two methods are more suitable for nonlinear systems with unknown dynamics. In this aspect, a large number of research results have been obtained [12-15]. Concrete results such as that in [16], a neural network-based adaptive FTC controller was proposed for the nonstrict-feedback interconnected large-scale systems, in which the neural networks were used to approximate the unknown terms of the considered systems. In [17], the fuzzy logic systems were presented to approximate the unknown terms, and then an adaptive fuzzy controller was designed by using backstepping technique, which ensured the finite-time convergence.

In addition, under the influence of environment and other factors, variables in the controlled system are often limited [18-20]; that is, variables need to be within a certain range and cannot be arbitrarily large. Therefore, constraint control has been widely concerned by researchers, and abundant achievements have been obtained [21-24]. In [25], the nonstrict feedback system with full-state constraints was considered, as well as input saturation and unmodeled dynamics. By introducing a logarithm nonlinear mapping, 
the considered system with full-state constraints was transformed into an unconstrained one.

According to the above analysis, an adaptive sliding mode controller with time-varying output constraint is designed. In order to ensure that the system output is with the specified time-varying constraint range, the asymmetric logarithmic Lyapunov function is selected. The unknown nonlinear function existed in the considered system is approximated by the radial basis function neural networks (RNFNNs). Then, an adaptive sliding mode controller is constructed by combining the backstepping technique and the sliding mode control approach.

Compared with the existing results, the innovations of this paper mainly include the following two aspects:

(1) Compared with the existing results in [21-25], where the constrained problems were considered and the symmetric barrier Lyapunov functions were employed, in this paper, the asymmetric barrier Lyapunov functions were used to solve the output constraint problem. Compared with symmetric barrier Lyapunov functions, asymmetric barrier Lyapunov functions are more widely used and less conservative.

(2) A new adaptive sliding mode control approach for manipulator systems is proposed by combining the barrier Lyapunov function method and the backstepping technique. A sufficient condition for the adaptive constrained control problem is derived. Moreover, the uncertainties are considered in this paper, which increase the design complexity.

The rest of this paper is organized as follows: Section 2 presents the problem description which includes the manipulator system description, the required assumption, and the control objective. Section 3 shows the design process of the adaptive NN controller, and the main result is also proposed. Section 4 contains the simulation example, and Section 5 is conclusion.

\section{Problem Description}

Consider the manipulator system in [26]

$$
\left\{\begin{array}{l}
\dot{x}_{1}=x_{2}, \\
\dot{x}_{2}=\frac{1}{M} x_{3}-\frac{N}{M} \sin x_{1}-\frac{B}{M} x_{2}, \\
\dot{x}_{3}=\frac{1}{L} u-\frac{K_{B}}{L} x_{2}-\frac{R}{L} x_{3}, \\
y=x_{1},
\end{array}\right.
$$

where $x_{1}, x_{2}, x_{3}$ are the system states, $u \in R$ and $y \in R$ are the control input and output, respectively, and the definitions of related notations can be found in [26]. In this paper, the system output is required to satisfy a certain constraint condition. That is to say the system output $y(t)$ satisfies the constraint condition that $\underline{k}(t) \leq y(t) \leq \bar{k}(t)$, where $\underline{k}(t)$ and $\bar{k}(t)$ are continuous functions.

Assumption 1 (see [27]). The desired reference signal $y_{d}(t)$ is bounded, which means that there exists a constant $\bar{Y}$ such that $\left|y_{d}(t)\right| \leq Y$.

The control target of this paper is to design an adaptive NN controller such that the closed-loop system is stable, and the system output can track the desired reference signal $y_{d}(t)$ as well as satisfy its constraint condition.

\section{The Adaptive NN Controller Design}

In this paper, the backstepping method is used to design the adaptive NN controller, and the following coordinate transformation is defined:

$$
\left\{\begin{array}{l}
z_{1}=x_{1}-y_{d}, \\
z_{2}=x_{2}-\alpha_{1}, \\
z_{3}=x_{3}-\alpha_{2},
\end{array}\right.
$$

where $z_{1}, z_{2}$, and $z_{3}$ are the error variables and $\alpha_{1}$ and $\alpha_{2}$ are the virtual controllers, which will be designed in the later procedure.

Step 1. From (1) and (2), one gets

$$
\begin{aligned}
\dot{z}_{1} & =\dot{x}_{1}-\dot{y}_{d} \\
& =z_{2}+\alpha_{1}-\dot{y}_{d} .
\end{aligned}
$$

Consider the following asymmetric obstacle Lyapunov function:

$$
V_{1}=\frac{q\left(z_{1}\right)}{2} \log \frac{k_{z_{1}}^{2}(t)}{k_{z_{1}}^{2}(t)-z_{1}^{2}}+\frac{1-q\left(z_{1}\right)}{2} \log \frac{k_{z_{2}}^{2}(t)}{k_{z_{2}}^{2}(t)-z_{1}^{2}},
$$

where $q\left(z_{1}\right)$ is a piecewise function, which means that if $z_{1}>0$, then $q\left(z_{1}\right)=1$, otherwise $q\left(z_{1}\right)=0 . k_{z_{1}}(t)$ and $k_{z_{2}}(t)$ are positive definite smooth bounded function, which satisfy $k_{z_{1}}(t)=\bar{k}(t)-Y$ and $k_{z_{2}}(t)=-\underline{k}(t)-Y$, respectively.

For convenience of expression, define the following error coordinate transformation:

$$
\left\{\begin{array}{l}
e_{1}=\frac{z_{1}}{k_{z_{1}}}, \\
e_{2}=\frac{z_{1}}{k_{z_{2}}}, \\
e=q\left(z_{1}\right) e_{1}+\left(1-q\left(z_{1}\right)\right) e_{2},
\end{array}\right.
$$

where $e_{1}, e_{2}$, and $e$ are transfer variables. Based on the above transformation, we know that if $z_{1}>0$, then $q\left(z_{1}\right)=1$ and $e=e_{1}$. Furthermore,

$$
V_{1}=\frac{1}{2} \log \frac{1}{1-e_{1}^{2}}=\frac{1}{2} \log \frac{1}{1-e^{2}} .
$$



have

Similarly, if $z_{1} \leq 0$, then $q\left(z_{1}\right)=0$ and $e=e_{2}$. Thus we

$$
V_{1}=\frac{1}{2} \log \frac{1}{1-e_{2}^{2}}=\frac{1}{2} \log \frac{1}{1-e^{2}} .
$$

Combining (6) and (7), one has

$$
V_{1}=\frac{1}{2} \log \frac{1}{1-e^{2}} \text {. }
$$

Then, the time derivative of $V_{1}$ is obtained as

$$
\begin{aligned}
\dot{V}_{1}= & \frac{q\left(z_{1}\right) e_{1}}{k_{z_{1}}\left(1-e_{1}^{2}\right)}\left(z_{2}+\alpha_{1}-\dot{y}_{d}-z_{1} \frac{\dot{k}_{z_{1}}}{k_{z_{1}}}\right)+\frac{\left(1-q\left(z_{1}\right)\right) e_{2}}{k_{z_{2}}\left(1-e_{2}^{2}\right)} \\
& \left(z_{2}+\alpha_{1}-\dot{y}_{d}-z_{1} \frac{\dot{k}_{z_{2}}}{k_{z_{2}}}\right) .
\end{aligned}
$$

Design the following virtual controller:

$$
\alpha_{1}=-\left(\gamma_{1}+\bar{\gamma}_{1}(t)\right) z_{1}+\dot{y}_{d}
$$

where $\quad \gamma_{1}>0$ is a constant and $\bar{\gamma}_{1}(t)=\sqrt{\left(\dot{k}_{z_{1}} / k_{z_{1}}\right)^{2}+\left(\dot{k}_{z_{2}} / k_{z_{2}}\right)^{2}+\gamma_{0}}$ is a time-varying gain with $\gamma_{0}$ being a positive constant.

Substituting (10) into (8), the following holds:

$$
\dot{V}_{1} \leq-\frac{\gamma_{1} e^{2}}{1-e^{2}}+\left(\frac{q\left(z_{1}\right)}{k_{z_{1}}^{2}-z_{1}^{2}}+\frac{1-q\left(z_{1}\right)}{k_{z_{2}}^{2}-z_{1}^{2}}\right) z_{1} z_{2} \leq-\frac{\gamma_{1} e^{2}}{1-e^{2}}+\mu z_{1} z_{2},
$$

where $\mu=\left(q\left(z_{1}\right) / k_{z_{1}}^{2}-z_{1}^{2}\right)+\left(1-q\left(z_{1}\right) / k_{z_{2}}^{2}-z_{1}^{2}\right)$.

Step 2. From (1) and (2), $\dot{z}_{2}$ can be described as

$$
\dot{z}_{2}=\frac{1}{M}\left(x_{3}-N \sin x_{1}-B x_{2}-M \dot{\alpha}_{1}\right) \text {. }
$$

In this paper, we consider the case that the load mass is uncertain, and then according to the definitions of the system parameter in [26], it is easy to know that $M$ and $N$ are unknown, which implies that the function $-N \sin x_{1}-B x_{2}-M \dot{\alpha}_{1}$ is also unknown. Here, the RBFNNs are used to approximate it, i.e.,

$$
-N \sin x_{1}-B x_{2}-M \dot{\alpha}_{1}=\omega_{2}^{* T} \varphi_{2}\left(X_{2}\right)+\varepsilon_{2}\left(X_{2}\right),
$$

where $\omega_{2}^{* T}$ stands for the ideal weight vector of the NNs, $\varphi_{2}\left(X_{2}\right)$ is a Gaussian function with $X_{2}=\left[x_{1}, x_{2}, x_{3}, y_{d}, \dot{y}_{d}\right]^{T}$ being the $\mathrm{NN}$ input, and $\varepsilon\left(X_{2}\right)$ is the approximation error. In addition, $\left\|\varepsilon_{2}\left(X_{2}\right)\right\| \leq \bar{\varepsilon}_{2}$.

Assumption 2 (see [28]). The ideal weight vectors of RBFNNs are bounded.

Substituting (13) into (12), one gets

$$
\dot{z}_{2}=\frac{1}{M}\left(x_{3}+\omega_{2}^{* T} \varphi_{2}\left(X_{2}\right)+\varepsilon_{2}\left(X_{2}\right)\right) .
$$

Select the following Lyapunov function:

$$
V_{2}=V_{1}+\frac{M}{2} z_{2}^{2}+\frac{1}{2 \delta_{2}} \tilde{W}_{2}^{2}
$$

where $\delta_{2}>0$ is a design parameter. $\widetilde{W}_{2}=W_{2}^{*}-\widehat{W}_{2}$ is the estimation error with $\widehat{W}_{2}$ being the estimation of $W_{2}^{*}$, and $W_{2}^{*}=\left\|\omega_{2}^{*}\right\|^{2}$.

Then, combining (11) and (14), the time derivative of $V_{2}$ is

$$
\begin{aligned}
\dot{V}_{2}= & \dot{V}_{1}+M z_{2} \dot{z}_{2}-\frac{1}{\delta_{2}} \widetilde{\omega}_{2}^{T} \dot{\hat{\omega}}_{2} \leq-\frac{\gamma_{1} e^{2}}{1-e^{2}}+\mu z_{1} z_{2} \\
& +z_{2}\left(x_{3}+\omega_{2}^{* T} \varphi_{2}\left(X_{2}\right)+\varepsilon_{2}\left(X_{2}\right)\right)-\frac{1}{\delta_{2}} \widetilde{W}_{2} \dot{\hat{W}}_{2} .
\end{aligned}
$$

Based on Young's inequality, it has

$$
z_{2} \omega_{2}^{* T} \varphi_{2}\left(X_{2}\right) \leq \frac{z_{2}^{2} W_{2}^{*} \varphi_{2}^{T}\left(X_{2}\right) \varphi_{2}\left(X_{2}\right)}{4 \xi_{2}}+\xi_{2} z_{2} \varepsilon_{2}\left(X_{2}\right) \leq \frac{1}{2} z_{2}^{2}+\frac{1}{2} \bar{\varepsilon}_{2}^{2}
$$

where $\xi_{2}>0$ is a parameter. Then, the derivative of $V_{2}$ becomes

$$
\dot{V}_{2} \leq-\frac{\gamma_{1} e^{2}}{1-e^{2}}+z_{2}\left(z_{3}+\alpha_{2}+\mu z_{1}+\frac{1}{2} z_{2}+\frac{z_{2} \widehat{W}_{2} \varphi_{2}^{T}\left(X_{2}\right) \varphi_{2}\left(X_{2}\right)}{4 \xi_{2}}\right)+\widetilde{W}_{2}\left(\frac{z_{2}^{2} \varphi_{2}^{T}\left(X_{2}\right) \varphi_{2}\left(X_{2}\right)}{4 \xi_{2}}-\frac{1}{\delta_{2}} \dot{\hat{W}}_{2}\right)+\frac{1}{2} \bar{\varepsilon}_{2}^{2}+\xi_{2} .
$$

Select the following virtual controller and adaptive law

$$
\begin{aligned}
\alpha_{2} & =-\gamma_{2} z_{2}-\mu z_{1}-\frac{1}{2} z_{2}-\frac{z_{2} \widehat{W}_{2} \varphi_{2}^{T}\left(X_{2}\right) \varphi_{2}\left(X_{2}\right)}{4 \xi_{2}}, \\
\dot{\hat{W}}_{2} & =\delta_{2}\left(\frac{z_{2}^{2} \varphi_{2}^{T}\left(X_{2}\right) \varphi_{2}\left(X_{2}\right)}{4 \xi_{2}}-\sigma_{2} \widehat{W}_{2}\right),
\end{aligned}
$$

where $\gamma_{2}$ and $\sigma_{2}$ are the positive constants.

Substituting (19) and (20) into $\dot{V}_{2}$, one has

$$
\begin{aligned}
\dot{V}_{2} & \leq-\frac{\gamma_{1} e^{2}}{1-e^{2}}-\gamma_{2} z_{2}^{2}+z_{2} z_{3}+\sigma_{2} \widetilde{W}_{2} \widehat{W}_{2}+\frac{1}{2} \bar{\varepsilon}_{2}^{2} \\
& \leq-\frac{\gamma_{1} e^{2}}{1-e^{2}}-\gamma_{2} z_{2}^{2}-\frac{1}{2} \sigma_{2} \widetilde{W}_{2}^{2}+z_{2} z_{3}+\frac{1}{2} \sigma_{2} W_{2}^{* 2}+\frac{1}{2} \bar{\varepsilon}_{2}^{2}+\xi_{2} .
\end{aligned}
$$

Step 3. In order to ensure that the tracking error of the system tends to zero in finite time, improve the convergence 
accuracy, and avoid singular problems; an integral end face sliding surface is designed as

$$
s=z_{3}+\eta \int_{0}^{t} z_{3}^{(l / n)} \mathrm{d} w
$$

where $\eta>0, l>0, n>0$, and $1<(l / n)<2$.

According to $z_{3}=x_{3}-\alpha_{2}$ and $\dot{x}_{3}=(1 / L) u-\left(K_{B} / L\right) x_{2}$ $-(R / L) x_{3}, \dot{s}$ can be obtained as

$$
\dot{s}=\frac{1}{L}\left(u-K_{B} x_{2}-R x_{3}-L \dot{\alpha}_{2}+L \eta z_{3}^{(l / n)}\right) .
$$

The function $-K_{B} x_{2}-R x_{3}-L \dot{\alpha}_{2}+L \eta z_{3}^{(l / n)}$ is unknown due to the unknown parameter $K_{B}$, and thus the RBFNNs are used to approximate it

$$
-K_{B} x_{2}-R x_{3}-L \dot{\alpha}_{2}+L \eta z_{3}^{(l / n)}=\omega_{3}^{* T} \varphi_{3}\left(X_{3}\right)+\varepsilon_{3}\left(X_{3}\right) .
$$

Therefore, $\dot{s}$ becomes

$$
\dot{s}=\frac{1}{L}\left(u+\omega_{3}^{* T} \varphi_{3}\left(X_{3}\right)+\varepsilon_{3}\left(X_{3}\right)\right) .
$$

Consider the Lyapunov function

$$
V_{3}=V_{2}+\frac{L}{2} s^{2}+\frac{1}{2 \delta_{3}} \tilde{W}_{3}^{2}
$$

where $\delta_{3}$ is a positive design parameter. $\widetilde{W}_{3}=W_{3}^{*}-\widehat{W}_{3}$ is the estimation error with $\widehat{W}_{3}$ being the estimation of $W_{3}^{*}$, and $W_{3}^{*}=\left\|\omega_{3}^{*}\right\|^{2}$.

Taking the derivative of $V_{3}$ along time, one has

$$
\dot{V}_{3}=\dot{V}_{2}+L s \dot{s}-\frac{1}{\delta_{3}} \widetilde{W}_{3} \dot{\widehat{W}}_{3} \text {. }
$$

Substituting (21) and (25) into (27), it has

$$
\begin{aligned}
\dot{V}_{3} \leq & -\frac{\gamma_{1} e^{2}}{1-e^{2}}-\gamma_{2} z_{2}^{2}-\frac{1}{2} \sigma_{2} \tilde{W}_{2}^{2}+z_{2} z_{3}+\frac{1}{2} \sigma_{2} W_{2}^{* 2}+\frac{1}{2} \bar{\varepsilon}_{2}^{2}+\xi_{2} \\
& -\frac{1}{\delta_{3}} \widetilde{W}_{3} \dot{\hat{W}}_{3}+s\left(u+\omega_{3}^{* T} \varphi_{3}\left(X_{3}\right)+\varepsilon_{3}\left(X_{3}\right)\right) .
\end{aligned}
$$

Using Young's inequality, we have

$$
s \omega_{3}^{* T} \varphi_{3}\left(X_{3}\right) \leq \frac{s^{2} W_{3} \varphi_{3}^{T}\left(X_{3}\right) \varphi_{3}\left(X_{3}\right)}{4 \xi_{3}}+\xi_{3} s \varepsilon_{3}\left(X_{3}\right) \leq \frac{1}{2} s^{2}+\frac{1}{2} \bar{\varepsilon}_{3}^{2},
$$

and then, we can deduce that

$$
\begin{aligned}
\dot{V}_{3} \leq & -\frac{\gamma_{1} e^{2}}{1-e^{2}}-\gamma_{2} z_{2}^{2}-\frac{1}{2} \sigma_{2} \tilde{W}_{2}^{2}+\frac{1}{2} \sigma_{2} W_{2}^{* 2}+\frac{1}{2} \bar{\varepsilon}_{2}^{2}+\frac{1}{2} \bar{\varepsilon}_{3}^{2}+s\left(u+\frac{s \widehat{W}_{3} \varphi_{3}^{T}\left(X_{3}\right) \varphi_{3}\left(X_{3}\right)}{4 \xi_{3}}+\frac{1}{2} s+\frac{z_{2} z_{3}}{s}\right) \\
& +\tilde{W}_{3}\left(\frac{s^{2} \varphi_{3}^{T}\left(X_{3}\right) \varphi_{3}\left(X_{3}\right)}{4 \xi_{3}}-\frac{1}{\delta_{3}} \dot{\widehat{W}}_{3}\right)+\xi_{2}+\xi_{3} .
\end{aligned}
$$

Design the following actual controller and adaptive law:

$$
\begin{aligned}
u & =-\gamma_{3} s-\frac{1}{2} s-\frac{z_{2} z_{3}}{s}-\frac{s \widehat{W}_{3} \varphi_{3}^{T}\left(X_{3}\right) \varphi_{3}\left(X_{3}\right)}{4 \xi_{3}}, \\
\dot{\hat{W}}_{3} & =\delta_{3}\left(\frac{s^{2} \varphi_{3}^{T}\left(X_{3}\right) \varphi_{3}\left(X_{3}\right)}{4 \xi_{3}}-\sigma_{3} \widehat{W}_{3}\right),
\end{aligned}
$$

where $\gamma_{3}$ and $\sigma_{3}$ are the positive parameters.

On the basis of (31) and (32), we get

$$
\dot{V}_{3} \leq-\frac{\gamma_{1} e^{2}}{1-e^{2}}-\gamma_{2} z_{2}^{2}-\gamma_{3} s^{2}-\frac{1}{2} \sum_{j=2}^{3} \sigma_{j} \tilde{W}_{j}^{2}+\frac{1}{2} \sum_{j=2}^{3} \sigma_{j} W_{j}^{* 2}+\frac{1}{2} \sum_{j=2}^{3}\left(\bar{\varepsilon}_{j}^{2}+\xi_{j}\right) \text {. }
$$

Let $\quad C=\min \left\{\gamma_{1}, \gamma_{2} / M, \gamma_{3} / L, \sigma_{2} \delta_{2}, \sigma_{3} \delta_{3}\right\}, \quad$ and $D=(1 / 2) \sum_{j=2}^{2}\left(\sigma_{j} \omega_{j}^{* T} \omega_{j}^{*}+\bar{\varepsilon}_{j}^{2}+\xi_{j}\right)$, from which $C$ and $D$ are the constants.
Furthermore, consider the inequality $-\left(\gamma_{1} e^{2} / 1-e^{2}\right) \leq-\gamma_{1} \log \left(1 / 1-e^{2}\right)$. Then, $\dot{V}_{3}$ can be described as 


$$
\dot{V}_{3} \leq-C V_{3}+D .
$$

Based on the above analysis, the following theorem is obtained.

Theorem 1. Consider the manipulator system (1); based on Assumptions 1-2, the controllers (10), (19), (31) and adaptive laws (20), (32) are designed such that all signals in the resulted system are bounded, the system output can track the reference signal to a compact set, and it does not violate its constraint.

Proof. . Select the Lyapunov function as follows:

$$
V=\frac{1}{2} \log \frac{1}{1-e^{2}}+\frac{M}{2} z_{2}^{2}+\frac{L}{2} s^{2}+\frac{1}{2} \sum_{j=2}^{3} \frac{1}{\delta_{j}} \widetilde{W}_{j}^{2}
$$

According to the above analysis, it is easily known that

$$
\dot{V} \leq-C V+D .
$$

The above inequality is integrated on both sides over $[0, t]$, and then the following holds:

$$
V(t) \leq V(0) e^{-C t}+\frac{D}{C} .
$$

According to the definition of $V$, we can know that $e, z_{2}$, $z_{3}, \widetilde{\omega}_{2}$, and $\widetilde{\omega}_{3}$ are all bounded for the bounded initial condition. When $z_{1}>0, e=e_{1}$, so $e_{1}$ is bounded. Since $e_{1}=$ $\left(z_{1} / k_{z_{1}}\right)$ and $k_{z_{1}}$ is a bounded function, $z_{1}$ is also bounded. Based on $z_{1}=x_{1}-y_{d}$ and the boundness of $y_{d}, x_{1}$ is bounded. According to the definition of $\alpha_{1}$, it is easy to know $\alpha_{1}$ is bounded. Furthermore, on the basis of $z_{2}=x_{2}-\alpha_{1}, x_{2}$ is also bounded. Similarly, $\alpha_{2}, x_{3}$, and $u$ are bounded. Thus, all signals in the resulted system are bounded.

In addition, from (35) and (36), one has

$$
\frac{1}{2} \log \frac{1}{1-e^{2}} \leq V(0) e^{-C t}+\frac{D}{C} .
$$

Furthermore, when $z_{1}>0, e=e_{1}=\left(z_{1} / k_{z_{1}}\right)$, and then

$$
z_{1} \leq k_{z_{1}} \sqrt{1-e^{-V(0) e^{-C t}-(D / C)}} .
$$

Similarly, when $z_{1} \leq 0$, we have

$$
z_{1} \leq k_{z_{2}} \sqrt{1-e^{-V(0) e^{-C t}-(D / C)}} .
$$

Therefore, when $t \longrightarrow \infty$, the tracking error $z_{1}$ converges to the compact set that

$$
z_{1} \leq k_{z} \sqrt{1-e^{-(D / C)}}
$$

where $k_{z}=\min \left\{k_{z_{1}}, k_{z_{2}}\right\}$. That is to say the system output can track the reference signal to a compact set.

Furthermore, from $z_{1}=x_{1}-y_{d}$, one has $-\left|z_{1}\right|-\left|y_{d}\right| \leq\left|x_{1}\right| \leq\left|z_{1}\right|+\left|y_{d}\right|$. As stated at the beginning of this paper, we can deduce that when $z_{1}>0, x_{1} \leq \bar{k}(t)$ and when $z_{1} \leq 0, x_{1} \geq \underline{k}(t)$. Thus, the system output satisfies the constraint condition.

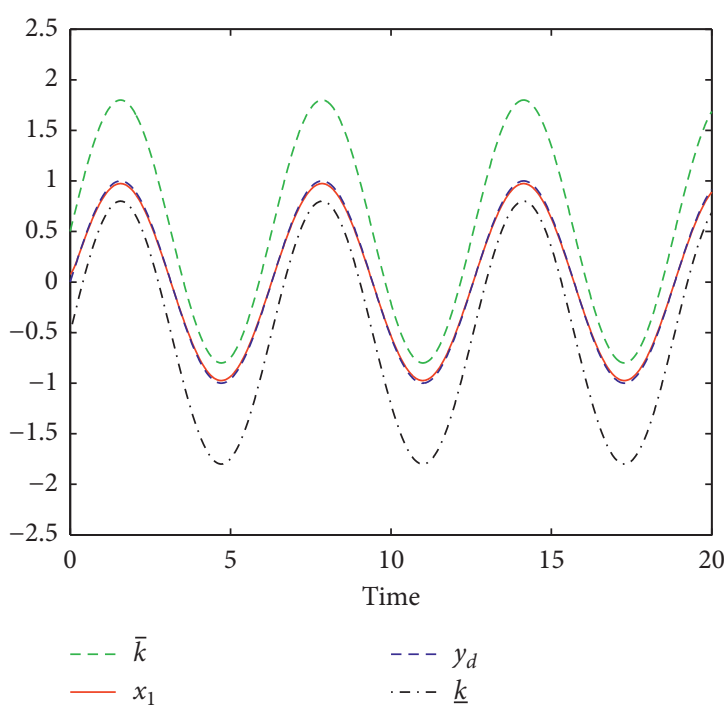

Figure 1: Trajectories of $x_{1}, y_{d}$ and the constraint bounds of $x_{1}$.

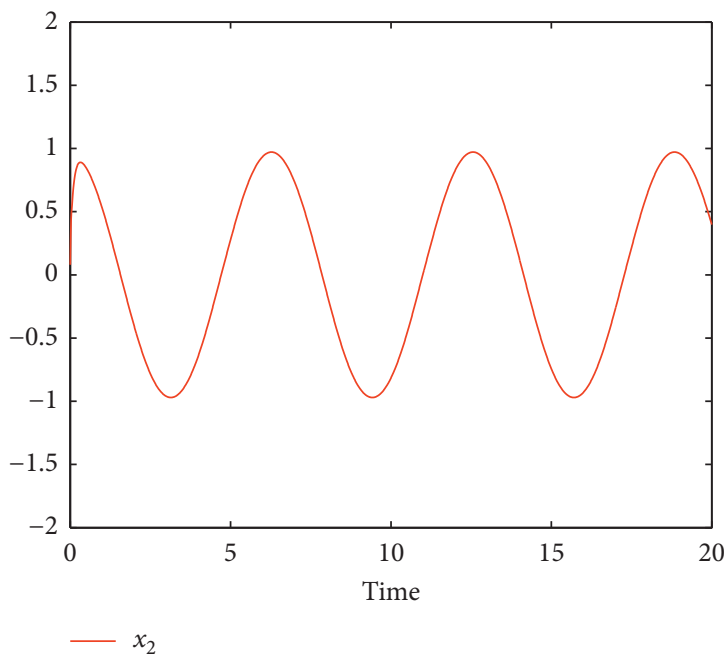

Figure 2: The trajectory of $x_{2}$.

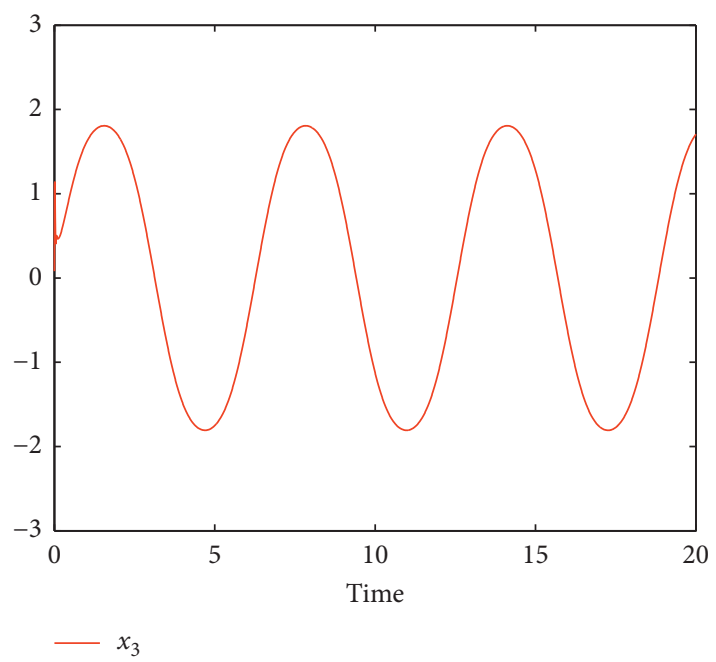

FIgURE 3: The trajectory of $x_{3}$. 


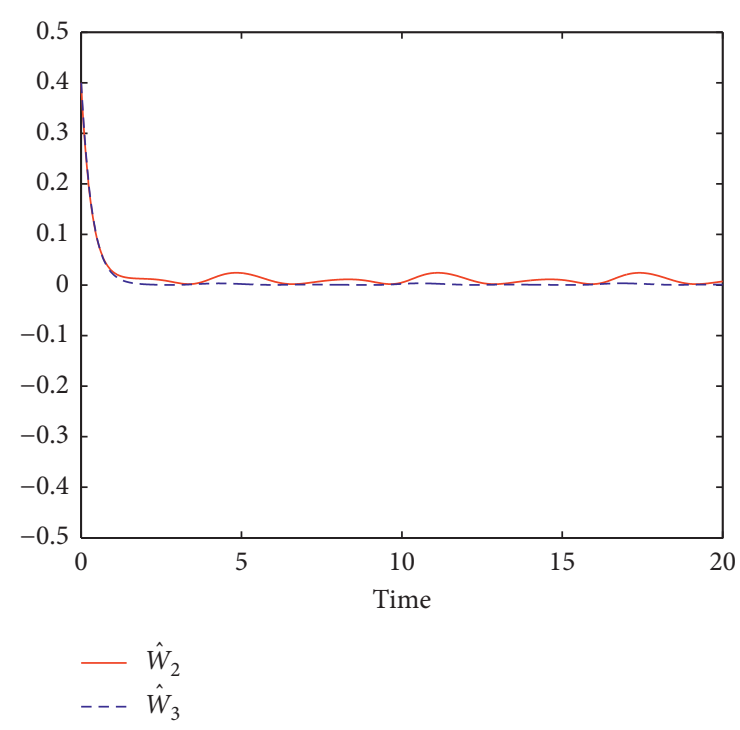

FIGURE 4: Trajectories of the update laws.

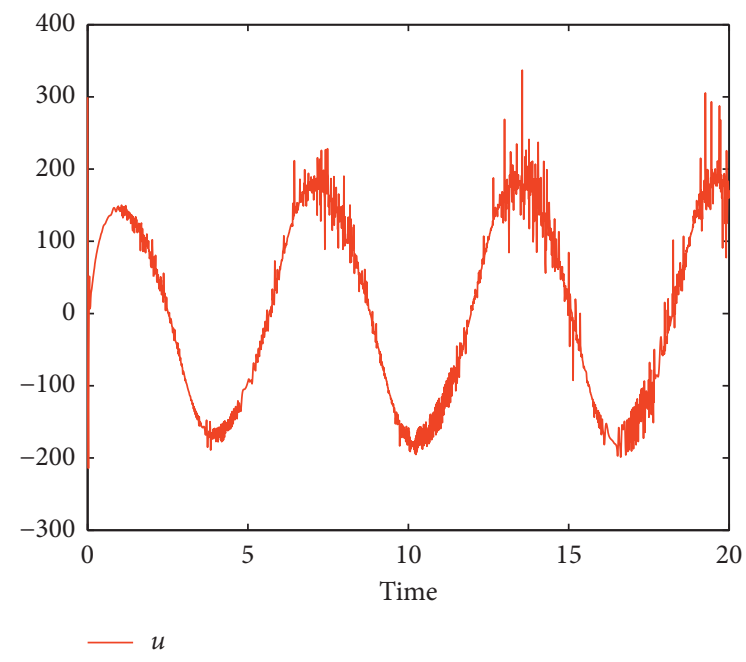

Figure 5: The trajectory of control input $u$.

This completes the proof of Theorem 1.

Remark 1. It is worth noting that equation (41) actually provides a direction on how to select some key design parameters: on the one hand, increasing $\gamma_{j}, j=1,2,3$, $\sigma_{j}, \quad j=2,3$, and $\delta_{j}, \quad j=2,3$ may help increase $C$, which further reduces $(D / C)$. On the other hand, decreasing $\xi_{j}, \quad j=2,3$, may help reduce $D$ and reduces $(D / C)$. The smaller $(D / C)$ leads to larger $e^{-(D / C)}$, which implies that the tracking error $z_{1}$ can be smaller, and then the tracking performance is better.

\section{Simulation Example}

In this section, the manipulator system (1) is considered, where

$$
\begin{aligned}
M & =\frac{J}{k_{\tau}}+\frac{m L_{0}^{2}}{3 k_{\tau}}+\frac{M_{0} L_{0}^{2}}{k_{\tau}}+\frac{2 M_{0} R_{0}^{2}}{5 k_{\tau}}, \\
B & =\frac{B_{0}}{k_{\tau}} \\
N & =\frac{m L_{0} G}{2 k_{\tau}}+\frac{M_{0} L_{0} G}{k_{\tau}}
\end{aligned}
$$

with $J=0.001625 \mathrm{~kg} \cdot \mathrm{m}^{2}, k_{\tau}=0.9 \mathrm{~N} \cdot \mathrm{m} / \mathrm{A}, m=0.506 \mathrm{~kg}$, $L_{0}=0.305 \mathrm{~m}, \quad M_{0}=0.434 \mathrm{~kg}, \quad R_{0}=0.022 \mathrm{~m}$, $B_{0}=0.01625 \mathrm{~N} \cdot \mathrm{m} \cdot \mathrm{s} / \mathrm{rad}$, and $G=9.8, L=0.225 \mathrm{H}$.

In this simulation, the desired signal is chosen as $y_{d}(t)=\sin t$, the constraint functions are $\hat{k}(t)=1.3 \sin (t)+0.5$ and $\underline{k}(t)=1.3 \sin (t)-0.5$. The initial values are selected as $x_{1}(0)=0.001, x_{2}(0)=0.012$, $x_{3}(0)=0.001, W_{2}(0)=0.01$, and $W_{3}(0)=1$, and the other related design parameters are chosen as $\gamma_{1}=8, \gamma_{2}=8$, $\gamma_{3}=22, \gamma_{0}=0.3, \delta_{2}=\delta_{3}=4, \sigma_{2}=\sigma_{3}=3$, and $\xi_{2}=\xi_{3}=1$.

Figures 1-5 show the simulation results of the adaptive sliding mode control strategy designed in this paper. Figure 1 describes the curves of system state $x_{1}$ (i.e., $y(t)$ ), the reference signal $y_{d}(t)$, and the constraint bounds of $x_{1}$. It can be seen that the system has a good tracking performance. At the same time, the output of the system is within the constraint range, i.e., it satisfies the constraint condition. Figures 2 and 3 show the trajectories of states $x_{2}$ and $x_{3}$, respectively. The adaptive laws for $\widehat{W}_{2}$ and $\widehat{W}_{3}$ are given in Figure 4 . According to Figures 2-4, it is easily known that they are bounded. Figure 5 expresses the trajectory of the control input $u$, which is also bounded. Thus, the effectiveness of the proposed method is well explained.

\section{Conclusion}

A sliding mode-based adaptive neural network control strategy is proposed for a manipulator system with output constraint. Combining the sliding mode control method with backstepping technique, an adaptive controller is designed, which not only solves the output constraint issue of the considered system, but also makes the error between system output and the reference signal to converge to a small compact set. Moreover, the output satisfies the constraint condition. Finally, the effectiveness of the proposed approach is illustrated by the simulation example.

\section{Data Availability}

The data used to support the findings of this study are included within the article.

\section{Conflicts of Interest}

The author declares no conflicts of interest. 


\section{References}

[1] V. M. Hernández-Guzmán, V. Santibáñez, and R. Campa, "PID control of robot manipulators equipped with brushless DC motors," Robotica, vol. 27, no. 2, pp. 225-233, 2009.

[2] V. M. Hernández-Guzmán and J. Orrante-Sakanassi, "Global PID control of robot manipulators equipped with PMSMs," Asian Journal of Control, vol. 20, no. 1, pp. 236-249, 2018.

[3] L. Liu and X. Li, "Event-triggered tracking control for active seat suspension systems with time-varying full state constraints," IEEE Transactions on Systems, Man, and Cybernetics: Systems, pp. 1-9, 2020.

[4] L. Tang and D. Li, "Time-varying barrier Lyapunov function based adaptive neural controller design for nonlinear purefeedback systems with unknown hysteresis," International Journal of Control, Automation and Systems, vol. 17, no. 7, pp. 1642-1654, 2019.

[5] L. Tang, A. Chen, and D. Li, “Time-varying tan-type barrier Lyapunov function-based adaptive fuzzy control for switched systems with unknown dead zone," IEEE Access, vol. 7, no. 1, pp. 110928-110935, 2019.

[6] J. Wang, W. Chen, Z. Chen et al., "Neural terminal slidingmode control for uncertain systems with building structure vibration," Complexity, vol. 2019, Article ID 507051, 9 pages, 2019.

[7] M. Van, "Adaptive neural integral sliding-mode control for tracking control of fully actuated uncertain surface vessels," International Journal of Robust and Nonlinear Control, vol. 29, no. 5, pp. 1537-1557, 2019.

[8] C. Huang, F. Naghdy, and H. Du, "Sliding mode predictive tracking control for uncertain steer-by-wire system," Control Engineering Practice, vol. 85, pp. 194-205, 2019.

[9] H. R. Nohooji, "Constrained neural adaptive PID control for robust manipulators," Journal of the Franklin Institute, vol. 357, no. 7, pp. 3907-3923, 2020.

[10] Y. Wang, X. Lai, P. Zhang, and M. Wu, "Adaptive robust control for planar n-link underactuated manipulator based on radial basis function neural network and online iterative correction method," Journal of the Franklin Institute, vol. 355, no. 17, pp. 8373-8391, 2018.

[11] S. Mishra, P. S. Londhe, S. Mohan, S. K. Vishvakarma, and B. M. Patre, "Robust task-space motion control of a mobile manipulator using a nonlinear control with an uncertainty estimator," Computers \& Electrical Engineering, vol. 67, pp. 729-740, 2018.

[12] L. Liu, Y.-J. Liu, and S. Tong, "Fuzzy-based multierror constraint control for switched nonlinear systems and its applications," IEEE Transactions on Fuzzy Systems, vol. 27, no. 8, pp. 1519-1531, 2019.

[13] L. Liu, X. Li, Y. J. Liu, and S. Tong, "Neural network based adaptive event trigger control for a class of electromagnetic suspension systems," Control Engineering Practice, vol. 106, Article ID 104675, 2021.

[14] Y. Liu, X. Liu, Y. Jing, and Z. Zhang, "A novel finite-time adaptive fuzzy tracking control scheme for nonstrict feedback systems," IEEE Transactions on Fuzzy Systems, vol. 27, no. 4, pp. 646-658, 2019.

[15] L. Tang and J. Zhao, "Switched threshold-based fault detection for switched nonlinear systems with its application to Chuas circuit system," IEEE Transactions on Circuits and Systems I: Regular Papers, vol. 66, no. 2, pp. 733-741, 2018.

[16] Y. Li and S. Tong, "Adaptive neural networks decentralized FTC design for nonstrict-feedback nonlinear interconnected large-scale systems against actuator faults," IEEE Transactions on Neural Networks and Learning Systems, vol. 28, no. 11, pp. 2541-2554, 2017.

[17] C.-W. Chang, C.-F. Hsu, and T.-T. Lee, "Backstepping-based finite-time adaptive fuzzy control of unknown nonlinear systems," International Journal of Fuzzy Systems, vol. 20, no. 8, pp. 2545-2555, 2018.

[18] W. Hu, C. Ruan, H. Nian, and D. Sun, "Zero-sequence current suppression strategy with common mode voltage control for open-end winding PMSM drives with common DC bus," IEEE Transactions on Industrial Electronics, p. 1, 2020.

[19] L. Liu, Y. J. Liu, S. C. Tong, and C. L. P. Chen, "Integral barrier Lyapunov function based adaptive control for switched nonlinear systems," Science China Information Sciences, vol. 63, no. 3, p. 132203, 2020.

[20] R. Wang, Q. Sun, P. Zhang et al., "Reduced-order transfer function model of the droop-controlled inverter via Jordan continued-fraction expansion," IEEE Transactions on Energy Conversion, vol. 35, no. 3, pp. 1585-1595, 2020.

[21] L. Wang and C. L. P. Chen, "Adaptive fuzzy dynamic surface control of nonlinear constrained systems with unknown virtual control coefficients," IEEE Transactions on Fuzzy Systems, vol. 28, no. 8, pp. 1737-1747, 2020.

[22] K. P. Tee and S. S. Ge, "Control of nonlinear systems with partial state constraints using a barrier Lyapunov function," International Journal of Control, vol. 84, no. 12, pp. 20082023, 2011.

[23] T. Gao, Y.-J. Liu, D. Li, S. Tong, and T. Li, "Adaptive neural control using tangent time-varying BLFs for a class of uncertain stochastic nonlinear systems with full state constraints," IEEE Transactions on Cybernetics, pp. 1-11, 2019.

[24] L. Wang, C. L. P. Chen, and H. Li, "Event-triggered adaptive control of saturated nonlinear systems with time-varying partial state constraints," IEEE Transactions on Cybernetics, vol. 50, no. 4, pp. 1485-1497, 2020.

[25] D. Ye, Y. Cai, H. Yang, and X. Zhao, "Adaptive neural-based control for non-strict feedback systems with full-state constraints and unmodeled dynamics," Nonlinear Dynamics, vol. 97, no. 1, pp. 715-732, 2019.

[26] D. M. Dawson, J. J. Carroll, and M. Schneider, "Integrator backstepping control of a brush DC motor turning a robotic load," IEEE Transactions on Control Systems Technology, vol. 2, no. 3, pp. 233-244, 1994.

[27] W. He, Y. Dong, and C. Sun, "Adaptive neural network control of unknown nonlinear affine systems with input deadzone and output constraint," ISA Transactions, vol. 58, pp. 96-104, 2015.

[28] D. J. Li, "Adaptive neural network control for a class of continuous stirred tank reactor systems," Science China Information Sciences, vol. 57, pp. 1-8, 2014. 